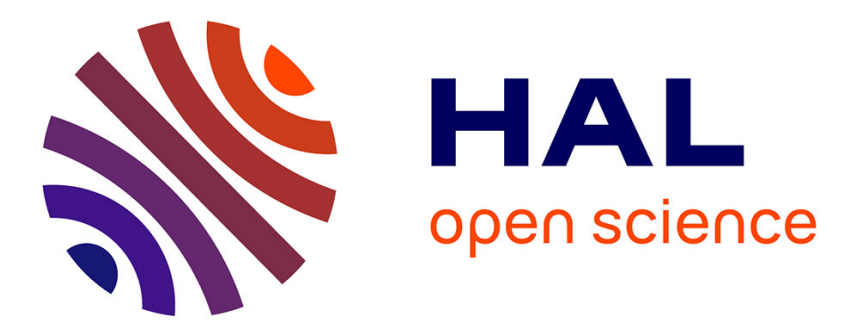

\title{
On the treatment of plane fusion front in lumped parameter thermal models with convection
}

\author{
R. Le Tellier, E. Skrzypek, L. Saas
}

\section{To cite this version:}

R. Le Tellier, E. Skrzypek, L. Saas. On the treatment of plane fusion front in lumped parameter thermal models with convection. Applied Thermal Engineering, 2017, 120, pp.314-326. 10.1016/j.applthermaleng.2017.03.108 . cea-02380935

\section{HAL Id: cea-02380935 https://hal-cea.archives-ouvertes.fr/cea-02380935}

Submitted on 26 Nov 2019

HAL is a multi-disciplinary open access archive for the deposit and dissemination of scientific research documents, whether they are published or not. The documents may come from teaching and research institutions in France or abroad, or from public or private research centers.
L'archive ouverte pluridisciplinaire HAL, est destinée au dépôt et à la diffusion de documents scientifiques de niveau recherche, publiés ou non, émanant des établissements d'enseignement et de recherche français ou étrangers, des laboratoires publics ou privés. 


\title{
On the treatment of plane fusion front in lumped parameter thermal models with convection
}

\author{
R. Le Tellier ${ }^{a, *}$ E. Skrzypek ${ }^{\mathrm{b}}$, L. Saas ${ }^{\mathrm{a}}$ \\ ${ }^{a} C E A, D E N, D T N / S M T A / L P M A$, Cadarache \\ F-13108 Saint Paul-lez-Durance, France \\ ${ }^{b}$ Institute of Heat Engineering, National Centre for Nuclear Research (NCBJ) \\ 05-400 Otwock-Świerk, Poland
}

\begin{abstract}
Within the framework of lumped parameter models for integral codes, this paper focus on the modelling of a two-phase Stefan fusion problem with natural convection in the liquid phase. In particular, this specific Stefan problem is of interest when studying corium pool behavior in the framework of light water reactor severe accident analysis. The objective of this research is to analyze the applicability of different approximations related to the modelling of the solid phase in terms of boundary heat flux closure relations. Three different approximations are considered: a quadratic profile based model, a model where a parameter controls the power partitioning at the interface and the steady state conduction assumption. These models are compared with an accurate fronttracking solution of this plane fusion front problem. This "reference" is obtained by combining the same integral conservation equations as the approximate models with a mesh-based solution of the 1D heat equation. Numerical results are discussed for a typical configuration of interest for corium pool analysis. Different fusion transients (constructed from nondimensionalization considerations in terms of Biot and Stefan numbers) are used in order to highlight the potential and limitations of the different approximations.
\end{abstract}

\footnotetext{
${ }^{*}$ Corresponding author

Email address: romain.le-tellier@cea.fr (R. Le Tellier)
}

Preprint submitted to International Journal of Heat and Mass Transfer September 21, 2016 
Keywords: plane fusion front, two-phase Stefan problem, lumped parameter model, focusing effect

\section{Research highlights:}

- Different solid phase approximations for a two-phase Stefan fusion problem with convection are analyzed.

- A "reference" solution is constructed by combining integral conservation equations and a mesh-based solution of the 1D heat equation.

- Numerical results are obtained for a typical configuration of interest in light water reactor severe accident analysis.

- The models performances are highlighted on different fusion transients constructed in terms of Biot and Stefan numbers.

\section{Introduction}

Mathematical and numerical description of solidification and melting of materials is a substantial issue arising in numerous engineering disciplines, including power and manufacturing engineering. For instance, in the context of nuclear power engineering, melting processes are of prime interest during a severe accident progression as a consequence of the insufficiency of the nuclear reactor core cooling. During this kind of event, the residual heat produced in the nuclear fuel coming from the fission products decay, is transferred depending on the system state and boundary conditions by convection, conduction or radiation across internal materials of the reactor pressure vessel.

For the special case of single pure material phase change, the moving boundary problem reduces to the so-called Stefan problem. In the Stefan problem, the moving phases interface and the associated conditions are non-linear with unknown location of the interface changing in time. The source of non-linearity lies in three aspects: the phase change itself at the interface, the properties of the material that are usually temperature dependent and lastly the boundary conditions of the domain that can be non-linear. This leads to a problem that is space and time dependent, which numerical solution aims at determining the interface position in the analysed domain (see, for instance, the review in [1] ). 
in which the behaviour of the material under various conditions is predicted. The problem was investigated in different ways that involved both analytical, numerical and experimental research.

In this paper, we are interested in the numerical simulation of such problems. 25 From this computational point of view, the different approaches found in the litterature can be classified into two broad categories: mesh-based discretizations of the local conservation equations (including fixed-grid and moving-grid methods) and lumped parameter models.

On the one hand, within the framework of mesh-based approaches, fixedgrid methods have received a lot of attention for their computational efficiency when $2 \mathrm{D}$ or $3 \mathrm{D}$ geometries are involved. The various methods differ in terms of the formulation for the energy conservation equation (see, for instance, the review in [2]). When convection in the liquid is to be taken into account, such methods becomes computationally demanding as the momentum conservation equation has to be solved; Computational Fluid Dynamics (CFD) tools are then used. For instance, in [3], CFD calculations were performed and compared with experimental results in order to validate the numerical approach based on the "Volume Of Fluid" method and an enthalpy formulation. In [4], a temperature transforming model is used in order to model melting with natural convection.

$40 \quad$ On the other hand, lumped parameter models for phase change problems are developed and used in so-called "0D" or integral codes that require fastrunning models, in particular when Monte-Carlo based sensitivity analyses are performed. In this context, one important case of interest is when the solid can be treated as one-dimensional. Many different approximations and simplifications have been proposed in this case (see [5] and citations therein). For instance, in [6], under the hypothesis that the Stefan number is lower than one, a quasi-static approximation was discussed and compared in [7] with CFD calculations with and without convection; a good agreement was reported. In [5], closures for the complete set of integral energy equations (liquid, solid and interface) are obtained based on Hermite approximations for integrals that define the average temperatures and boundary heat fluxes. Good agreement was shown 
with respect to both analytical formula for a semi-infinite medium without internal heat generation and a fixed-grid enthalpy method for internally heated slab configurations.

In this paper, the specific Stefan problem we are interested in is a twophase problem where convection in the liquid is taken into account through correlation-based closures and conduction in the solid can be approximated by the heat equation in a 1D slab. This specific Stefan problem is of interest when studying corium pool behavior in the framework of light water reactor severe accident analyses. In this context, integral models are used and the liquid phase modelling heavily relies on correlations obtained for natural convection in different configurations and for different regimes [8]. To the authors knowledge, a comprehensive analysis of the possible approximations of this Stefan problem from the point of view of solid conduction treatment is missing. This is the object of this paper where different approximations for the solid phase in such a lumped parameter modelling are compared in detail. We have considered different approximations that are in use in severe accident analysis comprising a quadratic profile based model (that is shown to be equivalent to the approach in [5]), a parametric model reported in [9] and the steady state conduction assumption (similar to [6]). The important feature of this comparison is that it is carried out with respect to a "reference" model that combines the same integral formulation of the conservation equations as the approximate models with a mesh-based solution of the 1D heat equation in the solid. In this way, an accurate front-tracking solution of this Stefan problem is obtained. It has the exact same modelling features as the approximate models under consideration except only for the solid boundary heat flux closure relations that are the object of our analysis.

The paper is structured as follows. Section 2 gives a brief presentation of the mathematical settings for the rest of the paper, both in terms of local and so macroscopic conservation equations. From there, the different models to be compared are discussed in Section 3, The "reference" model is given in Section 3.1 while Section 3.2 details the different approximate models under consideration. 
Numerical results and associated discussion are reported in Section 4 where a typical configuration of interest for light water reactor severe accident analysis is used along with nondimensionalization considerations (see Section 4.1) for constructing fusion transient cases. Finally, concluding remarks are given in Section 5.

\section{Mathematical settings}

This section briefly presents the mathematical settings of the physical model that represents a liquid domain $\Omega_{l}$ and a solid domain $\Omega_{s}$ separated by a sharp interface $\Gamma_{l s}$ associated with a plane fusion or solidification front. For the sake of clarity, the macroscopic balance equations are obtained from the local conservation equations and, then, they are simplified under the approximation of of a 1D slab solid.

\subsection{Local conservation equations}

The set of equations describing the system is composed of local conservation equations with the Stefan condition related to the moving interface.

For the liquid domain $\Omega_{l}(t)$, the mass, momentum and energy conservation equations are written under the Boussinesq approximation in terms of the liquid velocity $\vec{v}_{l}$ and the liquid temperature $T_{l}$ as:

$$
\begin{aligned}
& \vec{\nabla} \cdot \vec{v}_{l}(\vec{r}, t)=0 \text { for } \vec{r} \in \Omega_{l}(t) \\
& \frac{\partial \vec{v}_{l}}{\partial t}(\vec{r}, t)+\vec{v}_{l} \cdot \vec{\nabla} \vec{v}_{l}(\vec{r}, t)=\frac{-1}{\rho_{l}^{\mathrm{ref}}} \vec{\nabla} p(\vec{r}, t)+\nu_{l} \Delta \vec{v}_{l}(\vec{r}, t)-\beta_{l}\left(T_{l}(\vec{r}, t)-T_{l}^{\mathrm{ref}}\right) \vec{g} \\
& \text { for } \vec{r} \in \Omega_{l}(t) \\
& \rho_{l}^{\mathrm{ref}} C_{p, l}\left(\frac{\partial T_{l}}{\partial t}(\vec{r}, t)+\vec{v}_{l} \cdot \vec{\nabla} T_{l}(\vec{r}, t)\right)=\lambda_{l} \Delta T_{l}(\vec{r}, t) \text { for } \vec{r} \in \Omega_{l}(t) \\
& \vec{v}_{l}(\vec{r}, t) \cdot \vec{n}=0 \text { for } \vec{r} \in \partial \Omega_{l} \\
& \text { conditions in } T_{l}(\vec{r}, t) \text { and/or }-\lambda_{l} \vec{\nabla} T_{l}(\vec{r}, t) \cdot \vec{n} \text { for } \vec{r} \in \partial \Omega_{l}(t) \backslash \Gamma_{l s}(t) \\
& T_{l}(\vec{r}, t)=T^{\text {fus. }} \text { for } \vec{r} \in \Gamma_{l s}(t)
\end{aligned}
$$

with appropriate initial conditions (in particular, in terms of temperature $T_{l}(\vec{r}, 0)=$ $T_{l}^{0}(\vec{r})$ ). The liquid properties are: $\rho_{l}^{\text {ref }}$ the mass density at a reference tem- 
perature $T_{l}^{\text {ref }}, \nu_{l}$ the kinematic viscosity, $\beta_{l}$ the volumetric thermal expansion coefficient, $C_{p, l}$ the specific heat capacity and $\lambda_{l}$ the thermal conductivity.

In the solid domain $\Omega_{s}(t)$, the temperature $T_{s}$ is governed by the heat conduction equation:

$$
\begin{array}{rll}
\rho_{s} C_{p, s} \frac{\partial T_{s}}{\partial t}(\vec{r}, t)=\lambda_{s} \Delta T_{s}(\vec{r}, t) & \text { for } & \vec{r} \in \Omega_{s}(t) \\
\text { conditions in } T_{s}(\vec{r}, t) \text { and/or }-\lambda_{s} \vec{\nabla} T_{s}(\vec{r}, t) & \text { for } & \vec{r} \in \partial \Omega_{s} \backslash \Gamma_{l s}(t) \\
T_{s}(\vec{r}, t)=T^{\text {fus. }} & \text { for } & \vec{r} \in \Gamma_{l s}(t)
\end{array}
$$

with the initial condition $T_{s}(\vec{r}, 0)=T_{s}^{0}(\vec{r})$. The solid properties are: $\rho_{s}$ the mass density, $C_{p, s}$ the specific heat capacity and $\lambda_{s}$ the thermal conductivity.

Finally, the liquid-solid interface $\Gamma_{l s}$ velocity is described by a plane fusion front equation:

$$
\begin{array}{r}
\vec{v}^{\mathrm{ls}}(\vec{r}, t)=\frac{1}{\rho_{s} \Delta H^{\text {fus. }}}\left(-\lambda_{l} \vec{\nabla} T_{l}(\vec{r}, t) \cdot \vec{n}^{\mathrm{ls}}+\lambda_{s} \vec{\nabla} T_{s}(\vec{r}, t) \cdot \vec{n}^{\mathrm{ls}}\right) \vec{n}^{\mathrm{ls}} \\
\text { for } \vec{r} \in \Gamma_{l s}(t)
\end{array}
$$

with $\vec{n}^{\mathrm{ls}}$ is oriented from the liquid to the solid. The fusion properties are: $T^{\text {fus. }}$ the fusion temperature, $\Delta H^{\text {fus. }}$ the specific latent heat of fusion.

Note, that for the sake of conciseness and because of the selected applications in the numerical results section, no volumetric heat source has been introduced in these equations but the models presented hereafter are not restrictive with respect to this simplification.

\subsection{Lumped parameter formulation}

From there, letting aside the momentum equation in the liquid, the problem in both phases can be cast into an integral form in terms of macroscopic conservation equations for mass and energy. This approach leads to a so-called lumped parameter formulation (also called "0D" formulation).

In order to do so, the boundary $\partial \Omega_{l}(t)$ is assumed to be partitioned under the form $\partial \Omega_{l}(t)=\left(\bigcup_{i} \partial \Omega_{l, i}\right) \bigcup \Gamma_{l s}(t)$ and the following integral quantities are 
defined:

$$
\begin{aligned}
m_{l}(t) & \hat{=} \rho_{l} V_{l}(t) \\
\bar{T}_{l}(t) & \hat{=} \frac{1}{V_{l}(t)} \int_{\Omega_{l}(t)} T_{l}(\vec{r}, t) d V \\
\dot{m}^{\mathrm{ls}}(t) & \hat{=} \rho_{s} \int_{\Gamma_{l s}} \vec{v}^{\mathrm{ls}}(\vec{r}, t) \cdot \vec{n}^{\mathrm{ls}} d S \\
\bar{\phi}_{l}^{\mathrm{i}}(t) & \hat{=}-\frac{1}{S_{l}^{i}} \int_{\partial \Omega_{l, i}} \lambda_{l} \vec{\nabla} T_{l}(\vec{r}, t) \cdot \vec{n} d S \\
\bar{\phi}_{l}^{\mathrm{ls}}(t) & \hat{=}-\frac{1}{S_{l s}(t)} \int_{\Gamma_{l s}(t)} \lambda_{l} \vec{\nabla} T_{l}(\vec{r}, t) \cdot \vec{n}^{\mathrm{ls}} d S
\end{aligned}
$$

where $S_{l s}\left(\right.$ resp. $\left.S_{l}^{i}\right)$ is the area of $\Gamma_{l s}\left(\right.$ resp. $\left.\partial \Omega_{l, i}\right)$.

In this way, using Reynolds transport and Gauss theorem, the integral formulation of Eqs. 1 and 4 is obtained as:

$$
\begin{aligned}
\frac{d m_{l}(t)}{d t} & =\dot{m}^{\mathrm{ls}}(t) \\
C_{p, l}\left(m_{l}(t) \frac{d \bar{T}_{l}}{d t}(t)+\dot{m}^{\mathrm{ls}}(t)\left(\bar{T}_{l}(t)-T^{\mathrm{fus} .}\right)\right)= & -\sum_{i} \bar{\phi}_{l}^{\mathrm{i}}(t) S_{l}^{i} \\
& -\bar{\phi}_{l}^{\mathrm{ls}}(t) S_{l s}(t)
\end{aligned}
$$

The same integration process can be carried out for the solid domain to obtain an integral formulation as:

$$
\begin{aligned}
\frac{d m_{s}(t)}{d t}= & -\dot{m}^{\mathrm{ls}}(t) \\
C_{p, s}\left(m_{s}(t) \frac{d \bar{T}_{s}}{d t}(t)+\dot{m}^{\mathrm{ls}}(t)\left(T^{\mathrm{fus} .}-\bar{T}_{s}(t)\right)\right)= & -\sum_{i} \bar{\phi}_{s}^{\mathrm{i}}(t) S_{s}^{i} \\
& +\bar{\phi}_{s}^{\mathrm{ls}}(t) S_{l s}(t)
\end{aligned}
$$

with similar notations as for the liquid phase and the conductive heat flux defined as:

$$
\bar{\phi}_{s}^{\mathrm{ls}}(t) \hat{=}-\frac{1}{S_{l s}(t)} \int_{\Gamma_{l s}(t)} \lambda_{s} \vec{\nabla} T_{s}(\vec{r}, t) \cdot \vec{n}^{\mathrm{ls}} d S
$$

Finally, the interface Eq. 10 is written as:

$$
\dot{m}^{\mathrm{ls}}(t)=\frac{S_{l s}(t)}{\Delta H^{\text {fus. }}}\left(\bar{\phi}_{l}^{\mathrm{ls}}(t)-\bar{\phi}_{s}^{\mathrm{ls}}(t)\right)
$$


Obviously, the practical use of such 0D equations requires associated closure relations or Neumann boundary conditions associated with the average heat fluxes $\bar{\phi}_{l}^{\mathrm{i}}(t)$ and $\bar{\phi}_{s}^{\mathrm{i}}(t)$.

In the remainder, we will consider that adequate closure laws (i.e., in the form of Nusselt-Rayleigh correlations for natural convection) are available for the liquid phase 0D formulation associated with such a natural convection problem and we will focus our attention on the treatment of the solid phase.

\section{3. $1 D$ slab solid approximation}

In this study, the problem is further simplified under the hypothesis of a 1D slab fusion front as depicted in Figure 1. Note that the liquid shape is not specified except for its interface with the solid. The solid thickness $e_{s}(t)$ is given by $e_{s}=\frac{m_{s}}{S_{l s} \rho_{s}}$. The $\mathrm{x}$-axis origin is chosen on the external boundary of the liquid, the coordinate of the solid-liquid interface is given by $e_{l}(t)$ while the external boundary of the solid is at $x^{s}(t)=e_{l}(t)+e_{s}(t)$

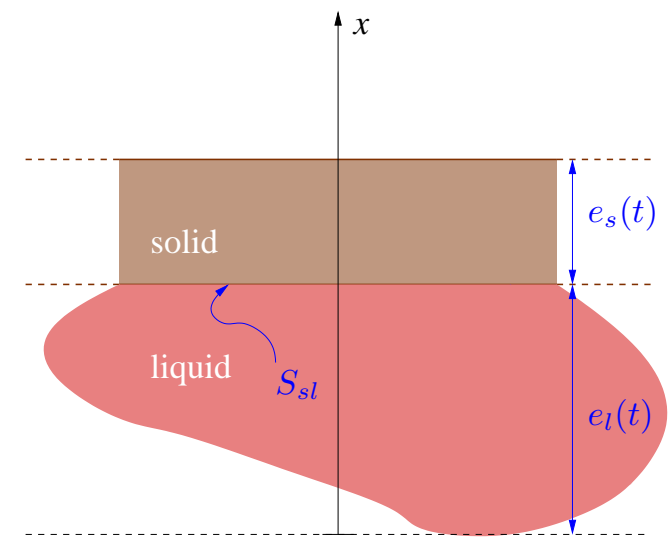

Figure 1: 1D front case and notations

In this case, the set of equations for the solid Eqs.7,8 and 9 can be simplified 
as:

$$
\begin{aligned}
& \rho_{s} C_{p, s} \frac{\partial T_{s}}{\partial t}(x, t)=\lambda_{s} \frac{\partial^{2} T_{s}}{\partial x^{2}}(x, t) \quad \text { for } \quad x \in\left[e_{l}(t), x^{s}(t)\right] \\
& \text { conditions in } T_{s}(x, t) \text { and/or }-\lambda_{s} \frac{\partial T_{s}}{\partial x}(x, t) \text { for } x=x^{s}(t) \\
& T_{s}(x, t)=T^{\text {fus. }} \quad \text { for } \quad x=e_{l}(t)
\end{aligned}
$$

In the same way, the lumped solid conservation equations Eq. 20 is:

$$
C_{p, s}\left(m_{s} \frac{d \bar{T}_{s}}{d t}+\dot{m}^{\mathrm{ls}}\left(T^{\mathrm{fus} .}-\bar{T}_{s}\right)\right)=\left(-\bar{\phi}_{s}^{\mathrm{bc}}+\bar{\phi}_{s}^{\mathrm{ls}}\right) S
$$

with $S=S_{l s}=S_{s}^{b c}$ and the average heat fluxes are:

$$
\begin{aligned}
\bar{\phi}_{s}^{\mathrm{ls}}(t) & =-\lambda_{s} \frac{\partial T_{s}}{\partial x}\left(e_{l}(t), t\right) \\
\bar{\phi}_{s}^{\mathrm{bc}}(t) & =-\lambda_{s} \frac{\partial T_{s}}{\partial x}\left(x^{s}(t), t\right)
\end{aligned}
$$

In the remainder, the system of equations to be solved will consist in the set of ordinary differential equations (ODE) composed of:

- the mass conservation equations for both liquid and solid phases Eqs. 17 and 19 coupled by the mass flow rate associated with the plane fusion front equation Eq. 21,

- the lumped liquid energy equation Eq. 18 with appropriate boundary conditions and/or closure laws on $\partial \Omega_{l}(t)$;

- the lumped solid energy equation Eq. 25] with heat fluxes $\bar{\phi}_{s}^{\mathrm{ls}}(t)$ and $\bar{\phi}_{s}^{\mathrm{bc}}(t)$ that are given by model-dependent equations discussed in the next section.

Note that these equations are valid only when both liquid and solid phases are present but, actually, the modelling presented here (and numerical results) also encompasses the cases when the system is completely liquid or solid. For the sake of conciseness, the equation modifications associated with these system states are not given as they are straightforward. The only additional difficulty is related to the transitions from one state to the other (appearance or disappearance of one phase) and its treatment in the numerical time integration; some explanations are given in Appendix A. 


\section{Front propagation models}

165 ODE system of equations with solid boundary heat fluxes $\bar{\phi}_{s}^{\mathrm{ls}}(t)$ and $\bar{\phi}_{s}^{\mathrm{bc}}(t)$.

\subsection{Reference model}

In order to compare different approximate models, a reference model has been constructed. A reference solution is obtained by solving explictly the 1D

170 heat equation of Eq. 22 (with boundary conditions given by Eqs. 23 and 24) in order to calculate the average heat fluxes $\bar{\phi}_{s}^{1 \mathrm{~s}}(t)$ and $\bar{\phi}_{s}^{\mathrm{bc}}(t)$. More precisely, the numerical scheme to treat the moving interface proceeds from time $t^{n}$ to time $t^{n+1}=t^{n}+\Delta t$ as follows:

- Eq. 22 is integrated from $t^{n}$ to $t^{n+1}$ based on an Euler implicit scheme i.e.

$$
\begin{aligned}
\rho_{s} C_{p, s} \frac{\tilde{T}_{s}\left(x, t^{n+1}\right)-T_{s}\left(x, t^{n}\right)}{\Delta t} & =\lambda_{s} \frac{\partial^{2} \tilde{T}_{s}}{\partial x^{2}}\left(x, t^{n+1}\right) \\
\text { for } \quad x & \in\left[e_{l}\left(t^{n}\right), x^{s}\left(t^{n}\right)\right]
\end{aligned}
$$

conditions in $\tilde{T}_{s}\left(x, t^{n+1}\right)$ and/or $-\lambda_{s} \frac{\partial T_{s}}{\partial x}\left(x, t^{n+1}\right)$ for $x=x^{s}\left(t^{n}\right)$

$$
\tilde{T}_{s}\left(x, t^{n+1}\right)=T^{\text {fus. }} \text { for } x=e_{l}\left(t^{n}\right)
$$

and a mesh-based discretization of this Laplacian equation.

- the ODE system is integrated from $t^{n}$ to $t^{n+1}$ considering that $\forall t \in$ $\left[t^{n}, t^{n+1}\right]$

$$
\begin{aligned}
\bar{\phi}_{s}^{\mathrm{ls}}(t) & =-\lambda_{s} \frac{\partial T_{s}}{\partial x}\left(e_{l}\left(t^{n}\right), t^{n+1}\right) \\
\bar{\phi}_{s}^{\mathrm{bc}}(t) & =-\lambda_{s} \frac{\partial T_{s}}{\partial x}\left(x^{s}\left(t^{n}\right), t^{n+1}\right)
\end{aligned}
$$

- from the previous integration, one obtains $e_{l}\left(t^{n+1}\right)$ that is used to update the mesh associated with the solid slab and calculate $T_{s}\left(x, t^{n+1}\right)$ by projection of $\tilde{T}_{s}\left(x, t^{n+1}\right)$ onto this new mesh over $\left[e_{l}\left(t^{n+1}\right), x^{s}\left(t^{n+1}\right)\right]$ and renormalization to ensure that $\int_{e_{l}\left(t^{n+1}\right)}^{x^{s}\left(t^{n+1}\right)} T_{s}\left(x, t^{n+1}\right) d x=e_{s}\left(t^{n+1}\right) \bar{T}_{s}\left(t^{n+1}\right)$. 
In practice, Eqs. 28, 29] and 30 are discretized using linear finite elements over a uniform mesh. Following [10], special care was simply taken in the evaluation of the heat fluxes at the boundary from this finite element solution in order to obtain superconvergent estimates for essential boundary conditions.

\subsection{Approximate models}

In this paper, the previous reference model is used to assess the behavior of different approximations of $\bar{\phi}_{s}^{\mathrm{ls}}(t)$ and $\bar{\phi}_{s}^{\mathrm{bc}}(t)$ that are used in integral models. In any case, these models give analytical closures for the ODE system.

\subsubsection{Quadratic profile based model}

A first approximate solution is constructed by replacing the direct discretization of Eq. 22 by a prescribed temperature profile $\tilde{T}_{s}(x, t)$ that satisfies the boundary conditions Eqs. 23 and 24 and preserve the average temperature $\bar{T}_{s}(t)$ i.e.

$$
\frac{1}{e_{s}} \int_{e_{l}}^{x^{s}} \tilde{T}_{s}(x, t) d x=\bar{T}_{s}(t)
$$

A simple adequate choice, completely defined by these three constraints, is to use a second-order polynomial as it is solution of the stationary 1D slab heat conduction equation with an internal source. Such an approximation is used in the MAAP source term code [11] or the PROCOR software platform [9] in the framework of light water reactor severe accident analysis. In this case, $\tilde{T}_{s}(x, t)$ is written as:

$$
\tilde{T}_{s}(x, t)=a\left(\frac{x^{s}-x}{e_{s}}\right)^{2}+b\left(\frac{x^{s}-x}{e_{s}}\right)+c
$$

with

$$
\begin{aligned}
c & =T_{s}\left(x^{s}, t\right) \\
b & =6 \bar{T}_{s}(t)-2 T^{\text {fus. }}-4 c \\
a & =3\left(\bar{T}_{s}(t)-\frac{b}{2}-c\right)
\end{aligned}
$$


The boundary heat fluxes are then given by

$$
\begin{aligned}
\bar{\phi}_{s}^{\mathrm{ls}}(t) & =\lambda_{s} \frac{2 a+b}{e^{s}}=-\lambda_{s} \frac{6 \bar{T}_{s}(t)-2 T_{s}\left(x^{s}, t\right)-4 T^{\text {fus. }}}{e^{s}} \\
\bar{\phi}_{s}^{\mathrm{bc}}(t) & =\lambda_{s} \frac{b}{e^{s}}=\lambda_{s} \frac{6 \bar{T}_{s}(t)-2 T^{\mathrm{fus} .}-4 T_{s}\left(x^{s}, t\right)}{e^{s}}
\end{aligned}
$$

Obviously if $\bar{T}_{s}(t)=\frac{T^{\text {fus. }}+T_{s}\left(x^{s}, t\right)}{2}$, a linear temperature profile is obtained i.e. $a=0$ in such a way that the steady state of the system without an internal source is correctly represented.

Actually, this approximation is equivalent to the one used in [5] and based on two-point Hermite-type approximations [12] for $T_{s}(x, t)$ and $\frac{\partial T_{s}}{\partial x}(x, t)$ integrals. More precisely, $\mathrm{H}^{1,1}\left(T_{s}\right)$ and $\mathrm{H}^{0,0}\left(\frac{\partial T_{s}}{\partial x}\right)$ (trapezoidal- rule) interpolation are used where, for any function $f$ defined over $\left[e_{l}, x^{s}\right], \mathrm{H}^{\nu, \mu}(f)$ denotes the Hermite interpolation based on values $\left(\frac{d^{i} f}{d x^{i}}\left(e_{l}\right)\right)_{0 \leq i \leq \nu}$ and $\left(\frac{d^{i} f}{d x^{i}}\left(x^{s}\right)\right)_{0 \leq i \leq \mu}$. In this way, the integral approximations are given by:

$$
\begin{aligned}
\bar{T}_{s}(t) & \approx \frac{1}{2}\left(T^{\text {fus. }}+T_{s}\left(x^{s}, t\right)\right)+\frac{e_{s}}{12}\left(\frac{\partial T_{s}}{\partial x}\left(e_{l}, t\right)-\frac{\partial T_{s}}{\partial x}\left(x^{s}, t\right)\right) \\
\int_{e_{l}}^{x^{s}} \frac{\partial T_{s}}{\partial x} d x & =T_{s}\left(x^{s}, t\right)-T^{\text {fus. }} \approx \frac{e_{s}}{2}\left(\frac{\partial T_{s}}{\partial x}\left(e_{l}, t\right)+\frac{\partial T_{s}}{\partial x}\left(x^{s}, t\right)\right)
\end{aligned}
$$

that are equivalent to Eqs. 38 and 39

\subsubsection{Parametric model}

In a second class approximation, when the steady-state of the system is known, inequalities relating the different heat fluxes can be obtained and may be used to construct a parametric approximation. For instance, let us consider a system in liquid-solid state under fusion for which it is known that, at final steady-state, the fusion is complete. Then, assuming that the fusion front monotonously propagates with a monotous evolution of the solid average temperature, while $\bar{T}_{s}<T^{\text {fus. }}$, the two following inequalities hold during all the fusion transient:

$$
\begin{aligned}
& \frac{d m_{l}}{d t} \geq 0 \\
& \frac{d \bar{T}_{s}}{d t} \geq 0
\end{aligned}
$$


Introducing these inequalities in Eqs. 25] and 21, the following inequalities on the heat fluxes are obtained:

$$
\begin{aligned}
\bar{\phi}_{s}^{\mathrm{ls}} & \leq \bar{\phi}_{l}^{\mathrm{ls}} \\
\bar{\phi}_{s}^{\mathrm{ls}} & \geq \frac{\bar{\phi}_{l}^{\mathrm{l}} S t_{s}+\bar{\phi}_{s}^{\mathrm{bc}}}{1+S t_{s}}
\end{aligned}
$$

where $S t_{s}=\frac{C_{p, s}\left(T^{\text {fus. }}-\bar{T}_{s}(t)\right)}{\Delta H^{\text {fus. }}}$.

Based on these relations, a parametric approximation of $\bar{\phi}_{s}^{\text {ls }}$ may be used under the form:

$$
\bar{\phi}_{s}^{\mathrm{ls}}=\left\{\begin{array}{rrr}
\min \left(\bar{\phi}_{l}^{\mathrm{ls}}, \omega \bar{\phi}_{l}^{\mathrm{ls}}+(1-\omega) \frac{\bar{\phi}_{l}^{\mathrm{ls}} S t_{s}+\bar{\phi}_{s}^{\mathrm{bc}}}{1+S t_{s}}\right) & \text { while } & \bar{T}_{s}<T^{\text {fus. }} \\
\bar{\phi}_{s}^{\mathrm{bc}} & \text { when } & \bar{T}_{s}=T^{\text {fus. }}
\end{array}\right.
$$

where $\omega$ is a dimensionless parameter $\in[0,1]$. For Neumann or Robin boundary conditions applied on the solid surface at $x^{s}$, this approximation of $\bar{\phi}_{s}^{\text {ls }}$ can be supplemented by an approximation of $\bar{\phi}_{s}^{\mathrm{bc}}$ or $T_{s}\left(x^{s}, t\right)$ in order to close the ODE system of equations for specific configurations. For the results presented in 230 $2 \bar{T}_{s}(t)-T^{\text {fus. }}$.

Such a parametric model may be useful in Monte-Carlo based sensitivity analyses considering that the treatment of the $\omega$ parameter as a random variable 
is a sound choice for capturing the overall model uncertainty. It was developed

in the PROCOR platform for this purpose [9].

\subsubsection{Steady-state conduction assumption}

As a last approximation, it is a common practice in "order of magnitude" evaluations such as in [13] to assume that the temperature profile in the solid is, at any time, the steady-state one i.e. a linear profile in such a way that

$$
\bar{\phi}_{s}^{\mathrm{ls}}(t)=\bar{\phi}_{s}^{\mathrm{bc}}(t)=-\lambda_{s} \frac{T_{s}\left(x^{s}, t\right)-T^{\text {fus. }}}{e^{s}}
$$

In this approximation, the solution of the lumped solid energy equation Eq.25]is no longer calculated as it is not needed. Note that with such an approximation, this equation would reduce to

$$
C_{p, s}\left(m_{s} \frac{d \bar{T}_{s}}{d t}+\dot{m}^{\text {ls }}\left(T^{\text {fus. }}-\bar{T}_{s}\right)\right)=0
$$

In the general case, the inconsistency between this energy balance and the steady-state linear profile approximation gives rather poor results (this discrepancy will be illustrated by numerical results in the next section). It is only

when the Stefan number is sufficiently small [6] (i.e. the second term in Eq. 48 is small) that this approximation is of interest.

\section{Numerical results}

In this section, the different approximations detailed in the previous sections will be compared with the reference model in order to discuss their shortcomings. This discussion on the models validity is not exhaustive and should not be taken as a general conclusion on the model performances. However, for the sake of genericity, the cases of study are selected with respect to a nondimensionalization of the equations presented hereafter.

\subsection{Nondimensionalization of the equations}

250 This nondimensionalization is limited to the front propagation (Eq. 21) and solid-related equations (Eqs. 22 and 25) as the treatment of the conduction in the solid in such a fusion problem is the focus of this work. 
The following notations are first introduced:

- scaling quantities: time $t_{\star}$, length $x_{\star}$, temperature $T_{\star}$, heat flux $\phi_{\star}$;

- dimensionless variables: $\tau=\frac{t}{t_{\star}}, \chi=\frac{x}{x_{\star}}, \chi^{s}=\frac{x^{s}}{x_{\star}}, \epsilon_{s}=\frac{e_{s}}{x_{\star}}, \epsilon_{l}=\frac{e_{l}}{x_{\star}}$, $\nu=\frac{t_{\star}}{x_{\star}} \frac{\dot{m}^{\mathrm{ls}}}{\rho_{s} S}, \theta=\frac{T_{s}-T^{\mathrm{fus}}}{T_{\star}}, \psi=\frac{\phi}{\phi_{\star}} ;$

- dimensionless numbers: Fourier number $F_{O}=\frac{\lambda_{s} t_{\star}}{\rho_{s} C_{p, s} x_{\star}^{2}}$, Biot number $B i=$ $\frac{\phi_{\star} x_{\star}}{\lambda_{s} T_{\star}}$, Stefan number $S t=\frac{C_{p, s} T_{\star}}{\Delta H^{\text {tuss }}}$.

In this way, the front equation Eq. 21 is written as:

$$
\nu=F o B i S t\left(\bar{\psi}_{l}^{\mathrm{ls}}-\bar{\psi}_{s}^{\mathrm{ls}}\right)
$$

The heat equation and associated boundary conditions Eqs. 22, 23 and 24 take the form:

$$
\begin{array}{rll}
\frac{\partial \theta}{\partial \tau}=F o \frac{\partial^{2} \theta}{\partial \chi^{2}} & \text { for } & \chi \in\left[\epsilon_{l}, \chi^{s}\right] \\
\text { conditions in } \theta(\chi, \tau) \text { and/or }-\frac{1}{B i} \frac{\partial \theta}{\partial \chi}(\chi, \tau) & \text { for } & \chi=\chi^{s} \\
\theta(\chi, \tau)=0 & \text { for } & \chi=\epsilon_{l}(t)
\end{array}
$$

Finally, the lumped solid energy conservation equation Eq. 25] is rewritten as:

$$
\left(\epsilon_{s} \frac{d \bar{\theta}}{d \tau}-\nu \bar{\theta}\right)=\operatorname{FoBi}\left(\bar{\psi}_{s}^{\mathrm{ls}}-\bar{\psi}_{s}^{\mathrm{bc}}\right)
$$

along with $\bar{\psi}_{s}^{\mathrm{ls}}=\frac{-1}{B i} \frac{\partial \theta}{\partial \chi}\left(\epsilon_{l}, \tau\right)$ and $\bar{\psi}_{s}^{\mathrm{bc}}=\frac{-1}{B i} \frac{\partial \theta}{\partial \chi}\left(\chi^{s}, \tau\right)$.

Then, the dimensionless time is taken as the heat conduction characteristic time in such a way that $F_{O}=1$ and the nondimensionalized equations only depends on $B i$ and $S t$ numbers.

As mentioned earlier, this nondimensionalization is incomplete as the coupling with the liquid equations (through $\bar{\psi}_{l}^{\mathrm{ls}}$ ) is not treated but it is still useful in a qualitative manner. Indeed, one can notice that the dimensionless front velocity $\nu$ is directly proportional to the product BiSt while the dimensionless heat fluxes are inversely proportional to $B i$; as a consequence, in the next section, the two following sensitivity analyses are considered: varying $B i$ while maintaining BiSt constant and varying $S t$ while maintaining $B i$ constant. 


\subsection{Cases of study}

The numerical results presented here are all based on the cylindrical geometry depicted in Figure 2 with a small aspect ratio (height over diameter). The system is heated from below with an imposed power $\bar{\phi}_{l}^{\text {in }} S$; Rayleigh-Benard convection in the liquid controls the power partitioning between the lateral boundary (heat flux $\bar{\phi}_{l}^{\text {lat }}$ and surface $S_{l}^{\text {lat }}$ ) and the solid/liquid interface. For these convective heat exchanges at the liquid boundaries, Nusselt-Rayleigh correlations are used: the Churchill \& Chu and Globe \& Dropkin correlations for the lateral and axial heat transfers respectively (see for instance [14] for more details on such closures for corium pool lumped models).

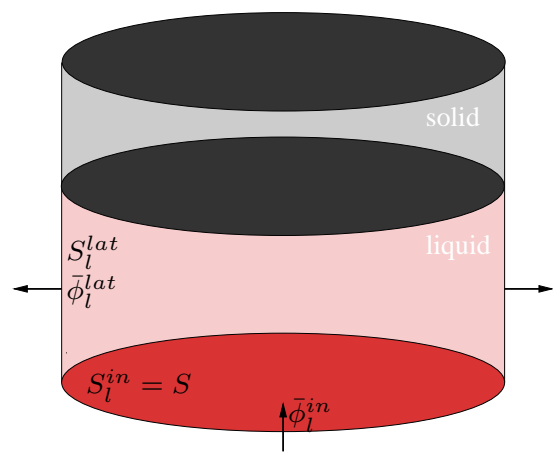

Figure 2: Geometrical configuration for the different cases and associated notations

A nominal test case was constructed on a typical configuration of interest for light water reactor severe accident analysis: within a vessel (the reactor vessel made of steel) a conductive material (steel coming from internal structures melting) is heated from below by a large volumetrically heated fluid pool (molten corium from the core meltdown) and cooled from above. The upper boundary condition associated with cooling is related to the availability of water to be injected on the system. While water is injected, the cooling provided by nucleate boiling at the surface is very efficient; when water is no longer available or if dryout occurs, the cooling is limited and the heat removal is mainly provided by radiative heat transfer. 
The simplified boundary conditions considered for this liquid/solid steel system are the following:

$$
\begin{array}{lll}
\text { on } & \partial \Omega_{l}^{i n}: & \text { imposed } \bar{\phi}_{l}^{\mathrm{in}} \\
\text { on } & \partial \Omega_{l}^{\text {lat }}: & T_{l}=T^{\mathrm{fus}} \\
\text { on } & \partial \Omega_{s}^{b c}: & \left\{\begin{array}{cl}
\bar{\phi}_{s}^{\mathrm{bc}}=h_{0}\left(T_{s}^{\mathrm{bc}}-T_{0}\right) & \text { for convective bc } \\
\bar{\phi}_{s}^{\mathrm{bc}}=\epsilon \sigma\left(\left(T_{s}^{\mathrm{bc}}\right)^{4}-\left(T_{0}\right)^{4}\right) & \text { for radiative bc }
\end{array}\right.
\end{array}
$$

with $T^{\text {fus }}$ the material liquidus temperature, $h_{0}$ a heat transfer coefficient representative of the heat exchange with water (nucleate boiling), $T_{0}$ the water saturation temperature and $\epsilon$ the emissivity of the solid surface. In order to consider the conditions on $\partial \Omega_{s}^{b c}$ in the reference model, the radiative condition has been linearized in such a way that Eq. 29 is written in any case as a Robin condition:

$$
-\lambda_{s} \frac{\partial T_{s}}{\partial x}\left(x, t^{n+1}\right)=h\left(T_{s}\left(x, t^{n+1}\right)-T_{0}\right) \quad \text { for } \quad x=x^{s}\left(t^{n}\right)
$$

with

$$
h=\left\{\begin{aligned}
h_{0} & \text { for convective bc } \\
\epsilon \sigma\left(T_{s}\left(x, t^{n}\right)+T_{0}\right)\left(\left(T_{s}\left(x, t^{n}\right)\right)^{2}+\left(T_{0}\right)^{2}\right) & \text { for radiative bc }
\end{aligned}\right.
$$

For the sake of simplicity, pure iron has been considered for the steel material. For the physical properties of solid and liquid phases, temperature-independent values were obtained from the PROCOR code 9] data (based on TOLBIAC-ICB code [15] subroutines) evaluated at $T^{\text {fus }}=1811 \mathrm{~K}$.

Then, the test case conditions are defined by the following:

- the cylinder radius is $1.4 \mathrm{~m}$ and the total mass of steel is fixed so that when the system is completely solid, its height is $e_{s}^{\max }=0.3 \mathrm{~m}$;

- at $t=0^{-}$, the system is at steady-state for a given $\bar{\phi}_{l}^{\mathrm{in}, 0}=0.4 \mathrm{MW} / \mathrm{m}^{2}$ and convective cooling on top with $h_{0}=10^{4} \mathrm{~W} / \mathrm{m}^{2} / \mathrm{K}, T_{0}=372.78 \mathrm{~K}$ in such a way that the system is composed of a solid phase on top of a liquid phase; 
- at $t=0^{+}$, the boundary conditions are modified as follows: $\bar{\phi}_{l}^{\text {in }}=3 \times \bar{\phi}_{l}^{\text {in, } 0}$ and convective heat transfer on the top boundary is replaced by radiative heat transfer.

The transient that follows leads to the complete fusion of the solid. In the context of severe accident analysis, the complete fusion of the solid is an important event because when the steel layer is completely liquid, the heat flux on its lateral boundary largely increases and becomes a major threat for the vessel integrity. This so-called "focusing effect" phenomenon (related to the high conductivity of this steel layer and its small aspect ratio) is of major concern in the evaluation of the in-vessel retention strategy where the aim is to contain the corium materials within the reactor pressure vessel (see, for instance, [16]).

Based on Section 4.1, the dimensionless numbers Bi and $S t$ were evaluated with $x_{\star}=e_{s}^{\max }, T_{\star}=T^{\text {fus }}-1000\left(1000 \mathrm{~K}\right.$ being an estimate of $\left.\bar{T}_{s}(t=0)\right)$ and $\phi_{\star}=\bar{\phi}_{l}^{\text {in, }, 0}$. From there, in addition to this nominal case, four additional tests 325 have been defined by modifying the solid physical properties:

- in two of these tests (denoted $\gamma_{\mathrm{Bi}}=0.5$ and $\gamma_{\mathrm{Bi}}=5.0$ ), both $\lambda_{s}$ and $\Delta H^{\text {fus. }}$ have been modified in order to decrease or increase $B i$ (multiplicative factor $\gamma_{\mathrm{Bi}}$ ) while maintaining BiSt constant. Note that the $B i$ value cannot be decreased much otherwise, the initial state (as given by the steady-state of the system for the conditions at $t=0^{-}$) and steady-state correspond to a completely solid steel layer.

- in the two other tests (denoted $\gamma_{\mathrm{St}}=0.1$ and $\gamma_{\mathrm{St}}=5.0$ ), $\Delta H^{\text {fus. }}$ has been modified in order to decrease or increase $S t$ (multiplicative factor $\gamma_{\mathrm{St}}$ ) while maintaining $B i$ constant.

These test cases and associated $B i, S t$ values are given in Table1 1 along with the initial solid thickness $e_{s}(0)$ and the time $t_{\mathrm{f}}$ at which the solid fusion is completed. 


\begin{tabular}{ccccc}
\hline case & $B i$ & $S t$ & $e_{s}(0)(\mathrm{m})$ & $t_{\mathrm{f}}(\mathrm{s})^{\dagger}$ \\
\hline nominal & 4.115 & 2.105 & 0.158 & 1371 \\
$\gamma_{\mathrm{Bi}}=0.5$ & 2.057 & 4.209 & 0.267 & 1721 \\
$\gamma_{\mathrm{Bi}}=5.0$ & 20.573 & 0.421 & 0.037 & 907 \\
$\gamma_{\mathrm{St}}=0.1$ & 4.115 & 0.201 & 0.158 & 6053 \\
$\gamma_{\mathrm{St}}=5.0$ & 4.115 & 10.523 & 0.158 & 974 \\
\hline
\end{tabular}

$\dagger$ as calculated with $\mathcal{R}\left(10^{-4}\right)$ model.

Table 1: Test cases description

The different models and associated parameters that are compared are summarized in Table 2. For the parametric model, while the $\omega$ parameter was varied from 0 to 1 with 0.1 increment, the results reported hereafter are limited to $\omega=0, \omega=1$ and the $\omega$ value giving the closest values to the $\mathcal{R}\left(10^{-4}\right)$ model.

\begin{tabular}{cl}
\hline notation & model \\
\hline $\mathcal{R}(\Delta x)$ & reference model with target mesh size $\Delta x \in\left\{10^{-4}, 10^{-3}\right\}$ (in m) \\
$\mathcal{Q}$ & quadratic profile-based approximate model \\
$\mathcal{P}(\omega)$ & parametric approximate model with parameter $\omega \in\{0, \ldots 1\}$ \\
$\mathcal{L}$ & steady-state linear profile-based approximate model \\
\hline
\end{tabular}

Table 2: Notations for the different models considered in the numerical tests.

For all models and all cases, the ODE system of equations is solved with an Euler explicit scheme with a prescribed timestep of $\delta t=0.1 \mathrm{~s}$. For the reference model, the timestep $\Delta t$ in the coupling scheme between the ODE system and the $1 \mathrm{D}$ conduction equation (see Section 3.1) is set to $\Delta t=5.0 \mathrm{~s}$ (except in the 
${ }_{345} \gamma_{\mathrm{Bi}}=0.5$ case, $\Delta t=2.5 \mathrm{~s}$ ). These values of $\delta t$ and $\Delta t$ are sufficiently small in order to ensure a proper model comparison (for the sake of conciness, the convergence with respect to these time discretizations is not shown here).

\subsection{Results and discussion}

For all cases, taking $\mathcal{R}\left(10^{-4}\right)$ result as the reference, for any other model

$\mathcal{M}$, the comparison is presented in terms of:

- the absolute error on the fusion time $\Delta t_{\mathrm{f}}=t_{\mathrm{f}}(\mathcal{M})-t_{\mathrm{f}}\left(\mathcal{R}\left(10^{-4}\right)\right)$;

- the relative error in $\mathrm{L}_{1}$-norm for any quantity $q \in\left\{\bar{\phi}_{s}^{\mathrm{ls}}, \bar{\phi}_{s}^{\mathrm{bc}}, \bar{T}_{s}, \bar{\phi}_{l}^{\mathrm{ls}}, e_{s}\right\}$ defined as:

$$
\varepsilon_{L_{1}}^{q}=\frac{\left\|q(\mathcal{M})-q\left(\mathcal{R}\left(10^{-4}\right)\right)\right\|_{1}}{\left\|q\left(\mathcal{R}\left(10^{-4}\right)\right)\right\|_{1}}
$$

with $\|\cdot\|_{1}$ calculated over the time interval $\left[0, \max \left(t_{\mathrm{f}}(\mathcal{M}), t_{\mathrm{f}}\left(\mathcal{R}\left(10^{-4}\right)\right)\right)\right]$.

These results for the nominal case are given in Table 3 where it can be seen that $\mathcal{R}\left(10^{-3}\right)$ results are very close to $\mathcal{R}\left(10^{-4}\right)$ demonstrating the convergence of this reference solution in terms of the spatial mesh size.

This nominal case gives a general view on the performances of the different approximate models for this typical configuration of interest in light water reactor severe accident analysis. The discrepancies of the $\mathcal{Q}$ model are limited, especially for the most important quantities $t_{\mathrm{f}}$ and $e_{s}$. Regarding the parametric model $\mathcal{P}$, while as expected the performances largely depend on the $\omega$ parameter, for the "optimal" $\omega$ value of 0.3 , the agreement on $t_{\mathrm{f}}$ and $e_{s}$ is comparable with the $\mathcal{Q}$ model but the error on the heat fluxes at the solid boundaries are larger. Finally, the steady-state conduction approximation appears, as expected, as a very poor approximation with $t_{\mathrm{f}}$ underestimated by about $50 \%$. 


\begin{tabular}{ccccccc}
\hline & $\Delta t_{\mathrm{f}}(\mathrm{s})$ & $\bar{\phi}_{s}^{\mathrm{ls}}$ & $\bar{\phi}_{s}^{\mathrm{bc}}$ & $\bar{T}_{s}$ & $\bar{\phi}_{l}^{\mathrm{ls}}$ & $e_{s}$ \\
\hline \hline $\mathcal{R}\left(10^{-3}\right)$ & 0.0 & 0.10 & 0.37 & 0.09 & 0.02 & 0.17 \\
\hline $\mathcal{Q}$ & -5.1 & 4.36 & 9.24 & 1.91 & 0.58 & 2.74 \\
\hline $\mathcal{P}(0.0)$ & -173.1 & 20.66 & 79.95 & 29.62 & 5.80 & 17.12 \\
$\mathcal{P}(0.3)$ & -5.1 & 6.46 & 30.62 & 2.59 & 0.86 & 3.39 \\
$\mathcal{P}(1.0)$ & 473.4 & 65.82 & 311.19 & 62.82 & 15.43 & 75.71 \\
\hline $\mathcal{L}$ & -698.1 & 80.44 & 175.67 & 206.02 & 20.42 & 56.47 \\
\hline
\end{tabular}

Table 3: Relative differences - nominal case - reference is $\mathcal{R}\left(10^{-4}\right)$.

For the sake of clarity, further discussion of the results in terms of model comparison is splitted into two parts: a comparative analysis between $\gamma_{\mathrm{Bi}}=$ 0.5 and $\gamma_{\mathrm{Bi}}=5.0$ cases in Section 4.3.1, $\gamma_{\mathrm{St}}=0.1$ and $\gamma_{\mathrm{St}}=5.0$ cases in Section 4.3.2. Finally, Section 4.3.3 provides additional results obtained on these transients in order to clearly highlight the shortcomings of the different approximate models.

\subsubsection{Varying Bi (maintaining BiSt constant)}

The results for $\gamma_{\mathrm{Bi}}=0.5$ and $\gamma_{\mathrm{Bi}}=5.0$ cases are presented in Tables 4 and 5 respectively in order to discuss the effect of increasing $B i$ (while maintaining BiSt constant).

Regarding the reference model, the discrepancy between $\mathcal{R}\left(10^{-3}\right)$ and $\mathcal{R}\left(10^{-4}\right)$ increases when $B i$ is increased: indeed, when the conductivity decreases, the transient thermal gradients within the solid are more pronounced and localized in such a way that a finer mesh at the boundary is needed.

Then, when $B i$ is increased, the "optimal" value of $\omega$ for the parametric model $\mathcal{P}$ decreases (from 0.4 to 0.1 when going from $\gamma_{\mathrm{Bi}}=0.5$ to $\gamma_{\mathrm{Bi}}=5.0$ ), while the $\mathcal{L}$ model results are largely improved. For both models, this trends can be related to the modification in the power partitioning between the solid heating and the fusion front propagation when $B i$ is varied. This variation of the power partitioning can be clearly illustrated by considering the following 
normalized quantity $\in[0,1]:$

$$
\Gamma_{\phi}^{\mathrm{ls}}=\frac{\bar{\phi}_{l}^{\mathrm{ls}}-\bar{\phi}_{s}^{\mathrm{ls}}}{\bar{\phi}_{l}^{\mathrm{ls}}-\bar{\phi}_{s}^{\mathrm{bc}}}
$$

that represents, for a given power input from the liquid at the interface and a given top boundary heat exchange, the fraction that is "used" for the front propagation. $\left(1-\Gamma_{\phi}^{\mathrm{ls}}\right)$ is then the fraction that goes into the solid heat balance (right hand side of Eq. 25). This quantity is depicted in Figure 3 for both $\gamma_{\mathrm{Bi}}=0.5$ and $\gamma_{\mathrm{Bi}}=5.0$ cases as calculated by the $\mathcal{R}\left(10^{-4}\right)$ model (note that, at $t=0^{+}$, due to the continuity of $\bar{\phi}_{l}^{\mathrm{ls}}$ and $\bar{\phi}_{s}^{\mathrm{ls}}, \Gamma_{\phi}^{\mathrm{ls}}$ is zero). It can be seen that, in average, $\Gamma_{\phi}^{\mathrm{ls}}$ becomes greater during the fusion transient when $B i$ is increased. This is consistent with the performance trends for the parametric and steadystate conduction models; indeed, with the parametric model, $\Gamma_{\phi}^{\mathrm{ls}}$ is maximum for $\omega=0$ while the steady-state conduction assumption gives $\Gamma_{\phi}^{\mathrm{ls}}=1$.

Finally, the $\mathcal{Q}$ model results are only slightly affected by $B i$ modification 390 and there is no specific trends (see Tables 3 , 4 and 5 ).

\begin{tabular}{ccccccc}
\hline & \multirow{2}{*}{$t_{\mathrm{f}}(\mathrm{s})$} & \multicolumn{5}{c}{$\varepsilon_{L_{1}}(\%)$} \\
& & $\bar{\phi}_{s}^{\mathrm{ls}}$ & $\bar{\phi}_{s}^{\mathrm{bc}}$ & $\bar{T}_{s}$ & $\bar{\phi}_{l}^{\mathrm{ls}}$ & $e_{s}$ \\
\hline \hline $\mathcal{R}\left(10^{-3}\right)$ & 0.0 & 0.06 & 0.26 & 0.07 & 0.03 & 0.12 \\
\hline $\mathcal{Q}$ & -0.3 & 2.68 & 7.46 & 1.75 & 0.86 & 3.07 \\
\hline $\mathcal{P}(0.0)$ & -182.0 & 17.60 & 79.74 & 27.51 & 6.84 & 18.24 \\
$\mathcal{P}(0.4)$ & -5.4 & 5.79 & 25.55 & 5.09 & 1.83 & 5.33 \\
$\mathcal{P}(1.0)$ & 241.9 & 46.77 & 220.86 & 36.87 & 17.19 & 62.68 \\
\hline $\mathcal{L}$ & -1115.4 & 87.68 & 169.55 & 214.66 & 29.10 & 70.44 \\
\hline
\end{tabular}

Table 4: Relative differences $-\gamma_{\mathrm{Bi}}=0.5$ case - reference is $\mathcal{R}\left(10^{-4}\right)$. 


\begin{tabular}{ccccccc}
\hline & \multirow{2}{*}{$t_{\mathrm{f}}(\mathrm{s})$} & $\bar{\phi}_{s}^{\mathrm{ls}}$ & $\bar{\phi}_{s}^{\mathrm{bc}}$ & $\bar{T}_{s}$ & $\bar{\phi}_{l}^{\mathrm{ls}}$ & $e_{s}$ \\
\hline \hline $\mathcal{R}\left(10^{-3}\right)$ & -5.0 & 1.01 & 2.11 & 0.77 & 0.20 & 0.18 \\
\hline $\mathcal{Q}$ & 0.1 & 4.41 & 2.82 & 0.64 & 0.04 & 0.77 \\
\hline $\mathcal{P}(0.0)$ & -163.1 & 48.32 & 86.55 & 38.65 & 6.52 & 18.96 \\
$\mathcal{P}(0.1)$ & -76.4 & 31.82 & 57.72 & 17.85 & 3.28 & 13.18 \\
$\mathcal{P}(1.0)$ & 651.9 & 193.88 & 416.76 & 102.70 & 15.86 & 86.18 \\
\hline $\mathcal{L}$ & -163.1 & 52.75 & 93.27 & 151.53 & 6.66 & 22.66 \\
\hline
\end{tabular}

Table 5: Relative differences $-\gamma_{\mathrm{Bi}}=5.0$ case - reference is $\mathcal{R}\left(10^{-4}\right)$.

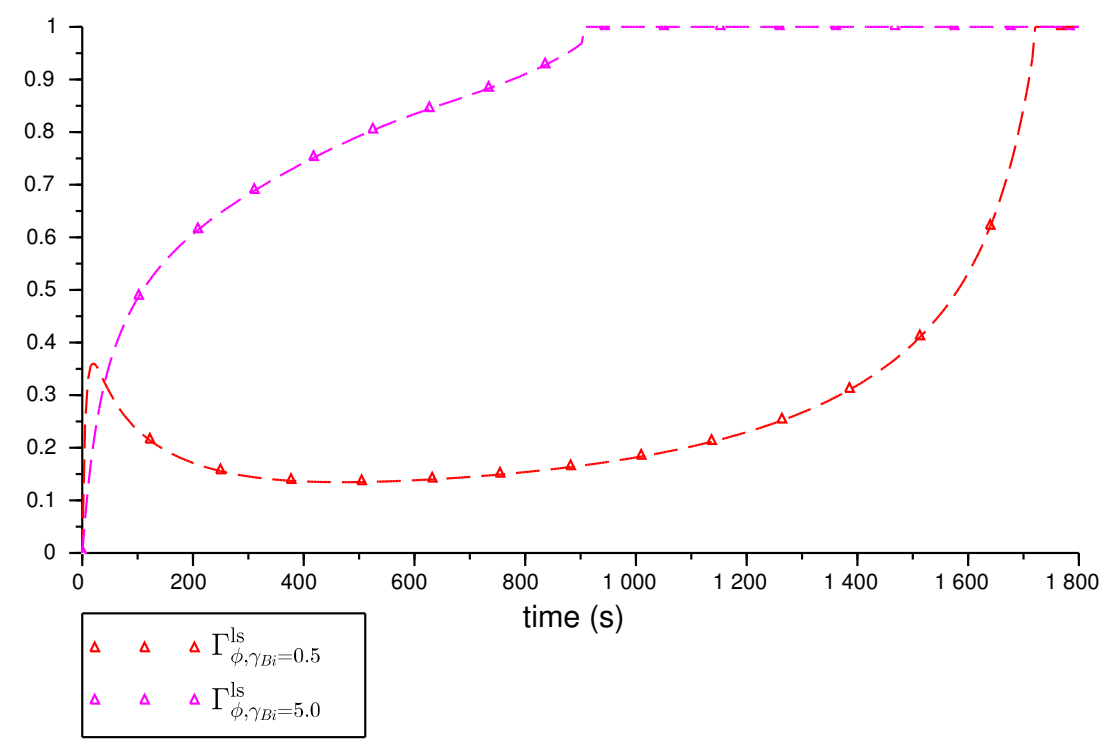

Figure 3: $\Gamma_{\phi}^{\mathrm{ls}}(t)-\gamma_{\mathrm{Bi}}=0.5$ and $\gamma_{\mathrm{Bi}}=5.0$ cases $-\mathcal{R}\left(10^{-4}\right)$ model

\subsubsection{Varying St (maintaining Bi constant)}

The results for $\gamma_{\mathrm{St}}=0.1$ and $\gamma_{\mathrm{St}}=5.0$ cases are presented in Tables 6 and 7 respectively in order to discuss the effect of increasing $S t$ (while maintaining $B i$ constant).

395

First of all, one can notice that the effect of refining the spatial mesh size from $10^{-3}$ to $10^{-4}$ is very limited and almost insensitive to the $S t$ value. 
Then, when $S t$ is increased, the "optimal" value of $\omega$ for the parametric model $\mathcal{P}$ increases (from 0.1 to 0.5 when going from $\gamma_{\mathrm{St}}=0.1$ to $\gamma_{\mathrm{St}}=5.0$ ), while the $\mathcal{L}$ model results are deteriorated. Alike previous cases where $B i$ was varied, this trend is related to the power partitioning between the solid heating and the fusion front propagation illustrated in Figure 4 in terms of $\Gamma_{\phi}^{\mathrm{ls}}(t)$ : when $S t$ increases, $\Gamma_{\phi}^{\mathrm{ls}}(t)$ is decreased. In addition, when $S t$ becomes small (i.e. for long fusion transient), the sensitivity of the parametric model results to the $\omega$ value becomes more important as can be seen from the error amplitude between $\omega=0$ and $\omega=1$ cases. While $\omega$ can still be adjusted to get results close to the reference (in the $\gamma_{\mathrm{St}}=0.1$ case, with $\omega=0.08$, the discrepancy on the fusion time is reduced to $39.7 \mathrm{~s}$ ), the constant power partitioning hypothesis of this model becomes inappropriate. This is further illustrated in Figure 5 where the $\bar{T}_{s}(t)$ evolution as calculated by $\mathcal{R}\left(10^{-4}\right)$ and $\mathcal{P}(0.1)$ is given. The evolution ${ }_{410}$ of $\bar{T}_{s}(t)$ can be separated around 1500 s into two time regions exhibiting very different slopes; as a consequence, in such a case, an "optimal" $\omega$ value is bound to underestimate (resp. overestimate) the slope of $\bar{T}_{s}(t)$ at the beginning (resp. end) of the fusion transient.

Finally, the $\mathcal{Q}$ model results are slightly deteriorated when $S t$ increases; the behavior of the $\mathcal{Q}$ model in the $\gamma_{\mathrm{St}}=5.0$ case is further discussed in Section 4.3 .3

\begin{tabular}{ccccccc}
\hline & \multirow{2}{*}{$t_{\mathrm{f}}(\mathrm{s})$} & $\bar{\phi}_{s}^{\mathrm{ls}}$ & $\bar{\phi}_{s}^{\mathrm{bc}}$ & $\begin{array}{c}\varepsilon_{L_{1}}(\%) \\
\bar{T}_{s}\end{array}$ & $\bar{\phi}_{l}^{\mathrm{ls}}$ & $e_{s}$ \\
\hline \hline $\mathcal{R}\left(10^{-3}\right)$ & 0.0 & 0.03 & 0.04 & 0.01 & 0.00 & 0.02 \\
\hline $\mathcal{Q}$ & 5.1 & 2.72 & 1.60 & 0.48 & 0.08 & 0.52 \\
\hline $\mathcal{P}(0.0)$ & -1382.1 & 65.90 & 80.57 & 45.34 & 7.65 & 19.49 \\
$\mathcal{P}(0.1)$ & 545.7 & 46.75 & 71.57 & 17.02 & 3.10 & 8.62 \\
$\mathcal{P}(1.0)$ & 3835.2 & 116.46 & 177.31 & 35.59 & 10.11 & 64.48 \\
\hline $\mathcal{L}$ & -601.8 & 37.97 & 48.72 & 279.75 & 4.07 & 14.90 \\
\hline
\end{tabular}

Table 6: Relative differences $-\gamma_{\mathrm{St}}=0.1$ case - reference is $\mathcal{R}\left(10^{-4}\right)$. 


\begin{tabular}{ccccccc}
\hline & \multirow{2}{*}{$t_{\mathrm{f}}(\mathrm{s})$} & $\bar{\phi}_{s}^{\mathrm{ls}}$ & $\bar{\phi}_{s}^{\mathrm{bc}}$ & $\begin{array}{c}\varepsilon_{L_{1}}(\%) \\
\bar{T}_{s}\end{array}$ & $\bar{\phi}_{l}^{\mathrm{ls}}$ & $e_{s}$ \\
\hline \hline $\mathcal{R}\left(10^{-3}\right)$ & 0.0 & 0.07 & 0.64 & 0.15 & 0.04 & 0.30 \\
\hline $\mathcal{Q}$ & -5.1 & 2.99 & 15.99 & 3.55 & 1.23 & 5.59 \\
\hline $\mathcal{P}(0.0)$ & -81.6 & 12.30 & 87.56 & 22.48 & 5.12 & 15.95 \\
$\mathcal{P}(0.5)$ & 5.1 & 3.90 & 35.69 & 8.26 & 2.15 & 13.26 \\
$\mathcal{P}(1.0)$ & 127.4 & 34.08 & 236.54 & 35.80 & 14.83 & 74.45 \\
\hline $\mathcal{L}$ & -749.6 & 92.75 & 159.52 & 146.72 & 29.77 & 80.11 \\
\hline
\end{tabular}

Table 7: Relative differences $-\gamma_{\mathrm{St}}=5.0$ case - reference is $\mathcal{R}\left(10^{-4}\right)$.

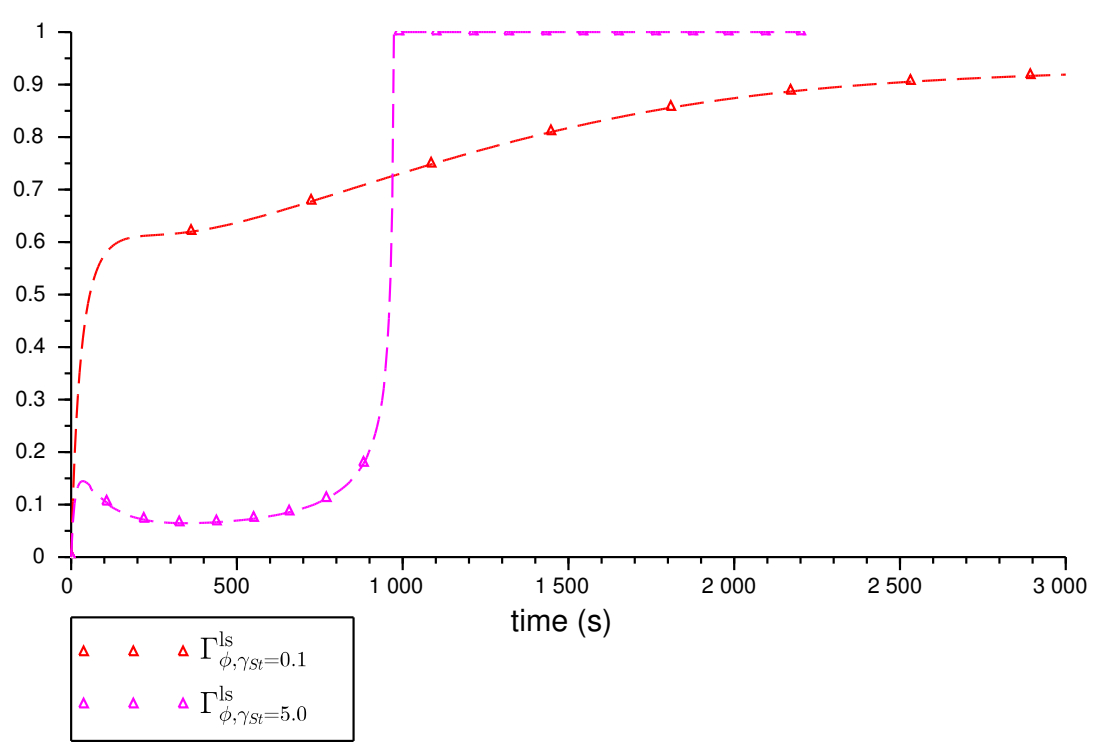

Figure 4: $\Gamma_{\phi}^{\mathrm{ls}}(t)-\gamma_{\mathrm{St}}=0.1$ and $\gamma_{\mathrm{St}}=5.0$ cases $-\mathcal{R}\left(10^{-4}\right)$ model. 


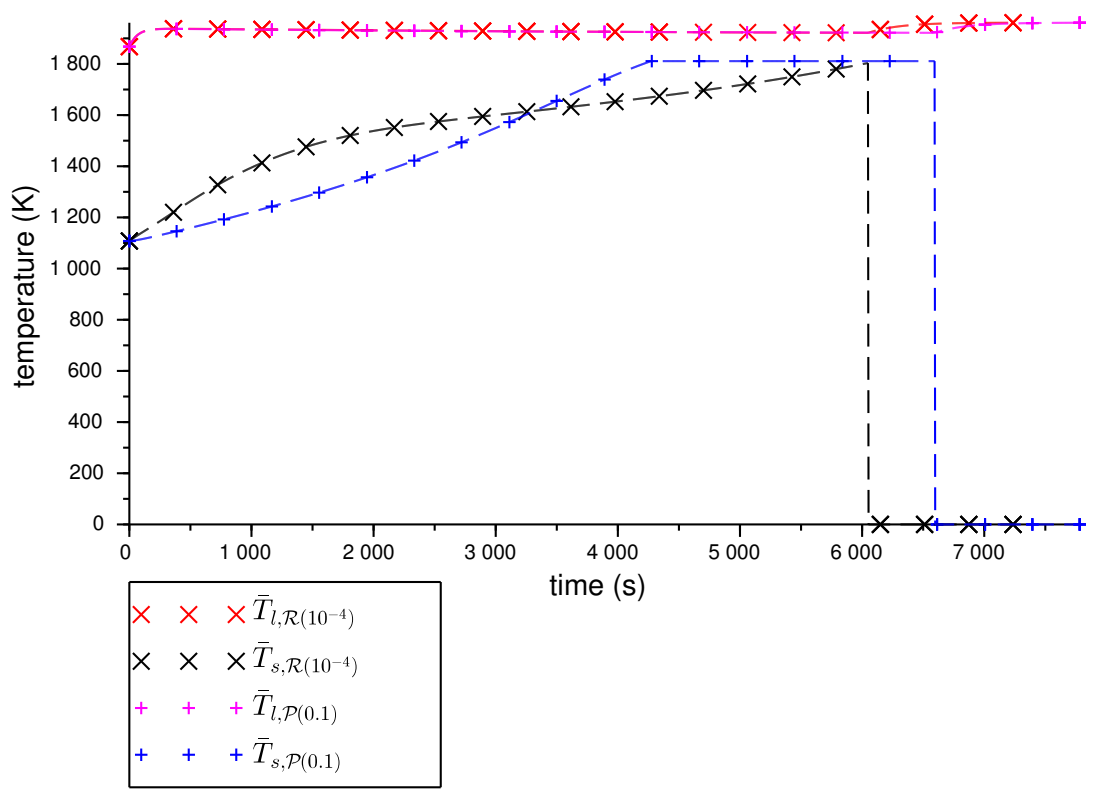

Figure 5: $\bar{T}_{s}(t)$ and $\bar{T}_{l}(t)-\gamma_{\mathrm{St}}=0.1-\mathcal{R}\left(10^{-4}\right)$ and $\mathcal{P}(0.1)$ models.

\subsubsection{Approximate models limitations}

To complete this analysis, the shortcomings of the different approximate models are illustrated in more detail by additional results.

First, an important feature of the $\mathcal{Q}$ model can be observed at the beginning of the transient: because of the quadratic profile asumption fitted on the average temperature $\bar{T}_{s}$ and both boundary conditions, the discontinuity at $t=0$ of the boundary condition on $\partial \Omega_{s}^{b c}$ leads to a non-physical discontinuity of the heat flux $\bar{\phi}_{s}^{\mathrm{ls}}$ at the solid-liquid interface as depicted in Figure 6 for the $\gamma_{\mathrm{St}}=5.0$ case. This discontinuity can induce a spurious behavior of the front propagation at the beginning of the transient. Indeed, as shown on Figure 7 for the $\gamma_{\mathrm{St}}=5.0$ case, for the first $16.6 \mathrm{~s}, \mathcal{Q}$ model predicts solidification at the interface instead of fusion: the $\mathcal{Q}$ model cannot ensure the correct direction of the front at all time.

Then, as explained in Section 3.2.3, an inherent feature of the $\mathcal{L}$ model is that the solid average temperature $\bar{T}_{s}$ decreases during a fusion transient. As 
shown in Figure 8 in the $\gamma_{\mathrm{Bi}}=0.5$, this decreasing trend can be very pronounced and leads to negative temperature that completely invalidate the transient solid heat balance with such a model.

Finally, as mentioned before, the parametric model performance is limited by the fixed value of $\omega$ that imposes a constant power partition at the interface. In addition, it is also inherently limited by the hypothesis $\frac{d \bar{T}_{s}}{d t} \geq 0$ that may not be always verified: as illustrated in Figure 9 in the $\gamma_{\mathrm{St}}=5.0$ case, the average temperature of the solid can slightly decreases at the beginning of a fast fusion transient.

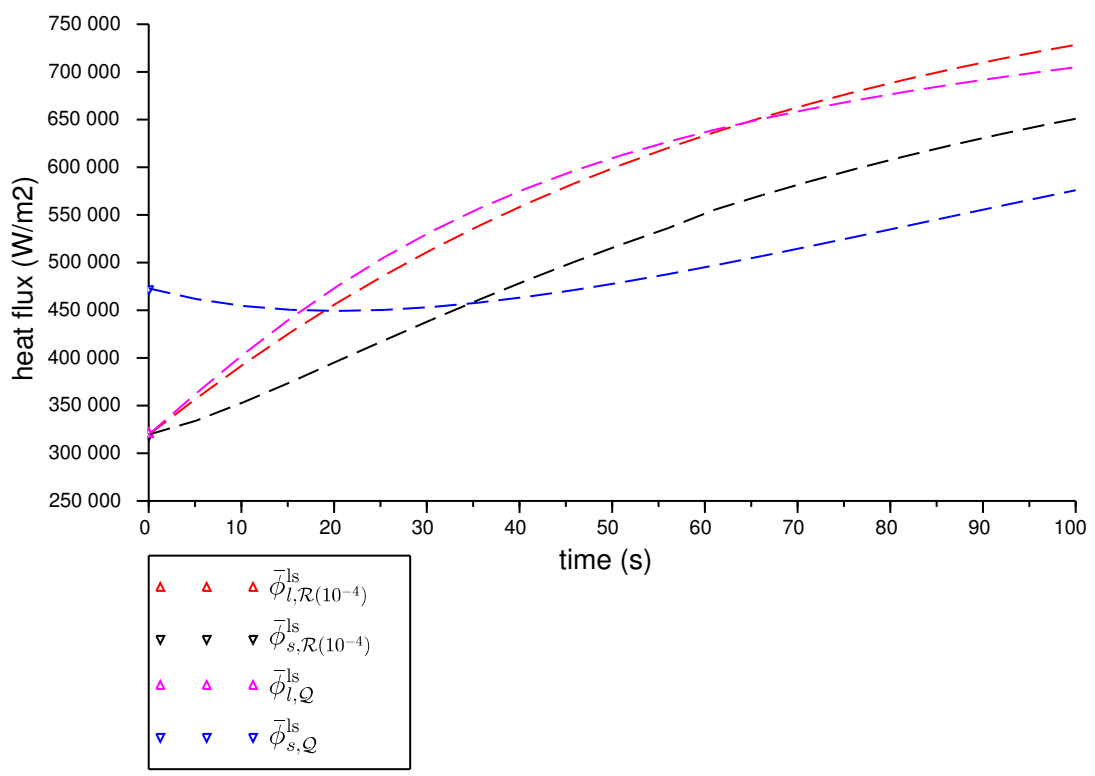

Figure $6: \bar{\phi}_{l}^{\mathrm{ls}}(t)$ and $\bar{\phi}_{s}^{\mathrm{ls}}(t)-\gamma_{\mathrm{St}}=5.0-\mathcal{R}\left(10^{-4}\right)$ and $\mathcal{Q}$ models. 


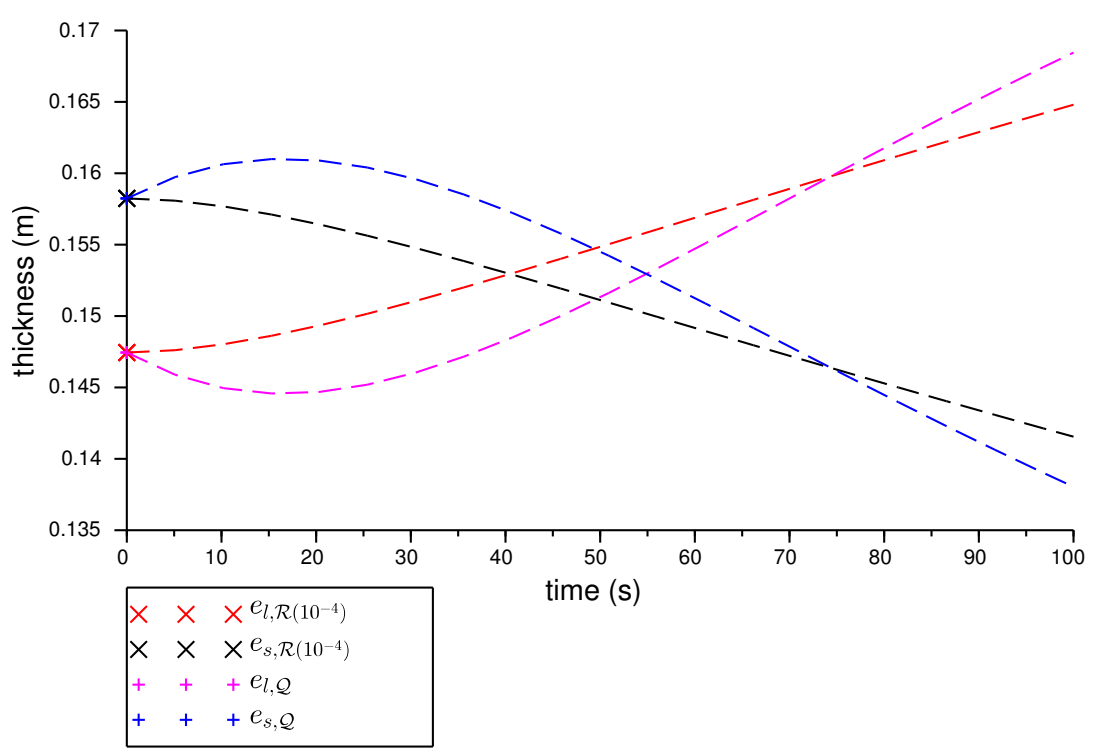

Figure 7: $e_{l}(t)$ and $e_{s}(t)-\gamma_{\mathrm{St}}=5.0-\mathcal{R}\left(10^{-4}\right)$ and $\mathcal{Q}$ models.

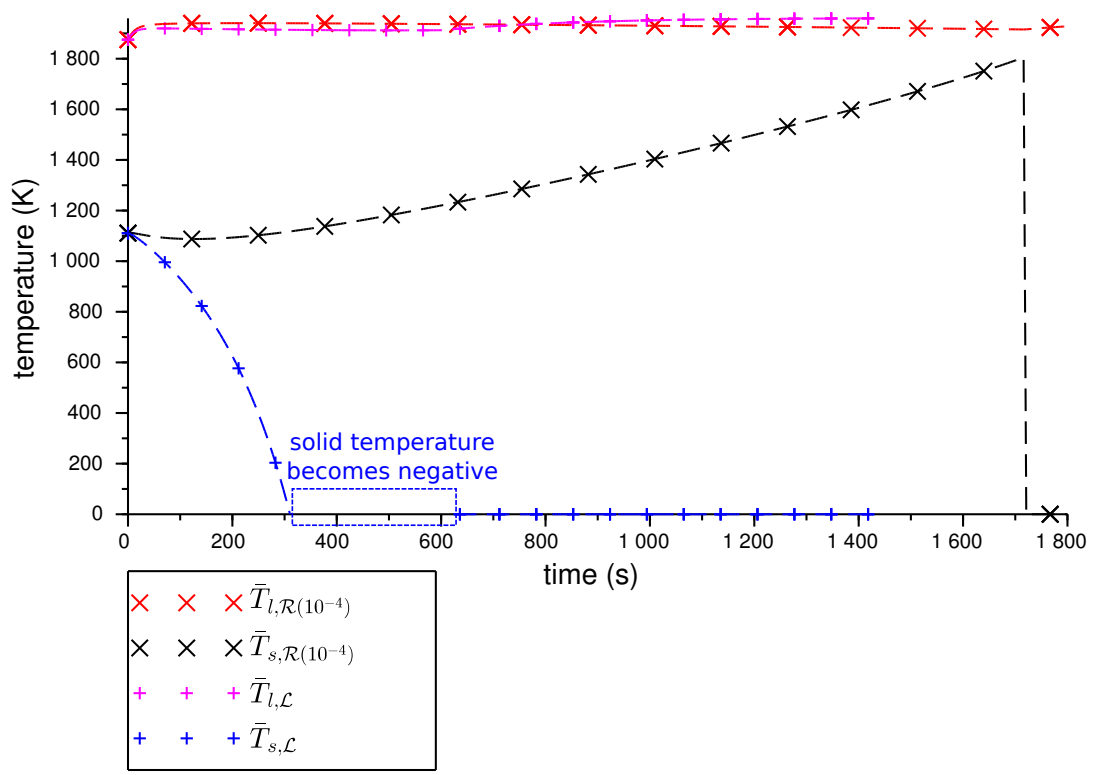

Figure 8: $\bar{T}_{l}(t)$ and $\bar{T}_{s}(t)-\gamma_{\mathrm{Bi}}=0.5-\mathcal{R}\left(10^{-4}\right)$ and $\mathcal{L}$ models. 


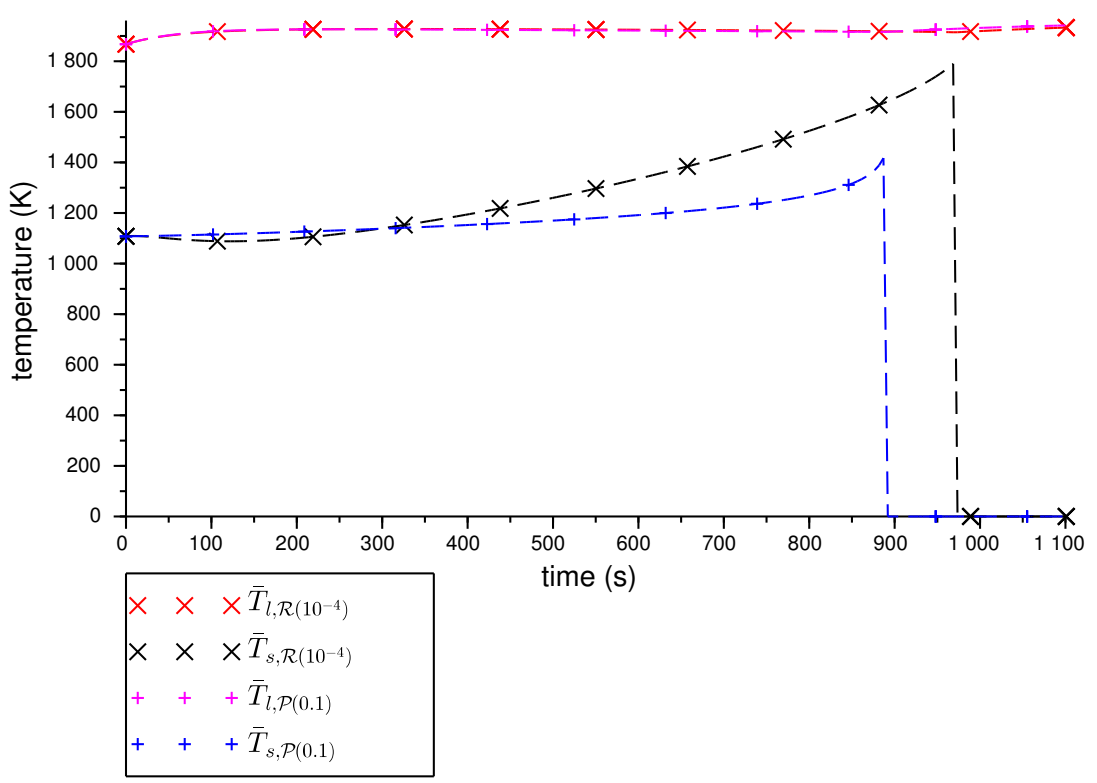

Figure 9: $\bar{T}_{l}(t)$ and $\bar{T}_{s}(t)-\gamma_{\mathrm{St}}=5.0-\mathcal{R}\left(10^{-4}\right)$ and $\mathcal{P}(0.1)$ models.

\section{Conclusion}

In this paper, the integral modelling of a two-phase Stefan problem with convection has been discussed from the point of view of solid boundary heat flux closure relations. The comparison of different approximate models has been carried out with respect to a "reference" model that combines the same integral formulation of the conservation equations as the approximate ones with a mesh-based solution of the $1 \mathrm{D}$ heat equation in the solid. Numerical results were discussed for fusion transients based on a typical configuration of interest for light water reactor severe accident analysis. A short parametric study based on nondimensionalization considerations in terms of Biot and Stefan numbers was presented in order to highlight the limitations of the different approximations. On the overall, the quadratic profile based model (equivalent to two-point Hermite approximations for the integrals that define the average temperatures and boundary heat fluxes) appears as a good approximation while the applicability of the parametric model and the steady state conduction assumption is shown 
to be limited to certain fusion transients depending on the power partitioning at the liquid-solid interface between the front propagation and the solid heating.

\section{Acknowledgments}

This work has been carried out within the framework of the PROCOR platform development funded by CEA, EDF and AREVA.

\section{Appendix A. ODE system numerical integration}

The ODE systems are solved using the Apache Commons Math library [17] in the JAVA language. A set of additional top-level classes have been added in the PROCOR platform (kernel package) in order to facilitate the description of such ODE systems in the context of lumped mass and energy conservation equations. In particular, based on the discrete event handling tools of this library (see Apache Commons Math ode package), a model can explicitly declare the different states of its underlying ODEs in such a way that the detection and treatment of the associated transitions can be taken care of properly during the integration process. The state transitions are described through "switching" functions (see Section 6.3 in [18] for instance) and, through a root-finding algorithm, these functions are used within the time integration process iin order to refine the time step (independently of the time integration scheme) in order to adequately capture the state transitions. Using this library and this formalism, many different integration methods can be used in a transparent way (in this study, an explicit Euler scheme was used).

For our fusion/solidification front ODE model, five different states and associated transitions have been distinguished as shown in Figure A.10 that also depicts the transition conditions in terms of mass and temperature thresholds. 480 In addition to the three states "liquid only", "liquid-solid" and "solid only", two additional intermediate states have been introduced as transitional states in order to stabilize the front propagation and avoid numerical problems: when a phase appears (surface temperature reaches $T^{\text {fus }}$ ), in these intermediate states, 
the appearing phase is supposed to remain constant at $T^{\text {fus }}$ in such a way that

only the mass conservation equation is integrated for this appearing phase.

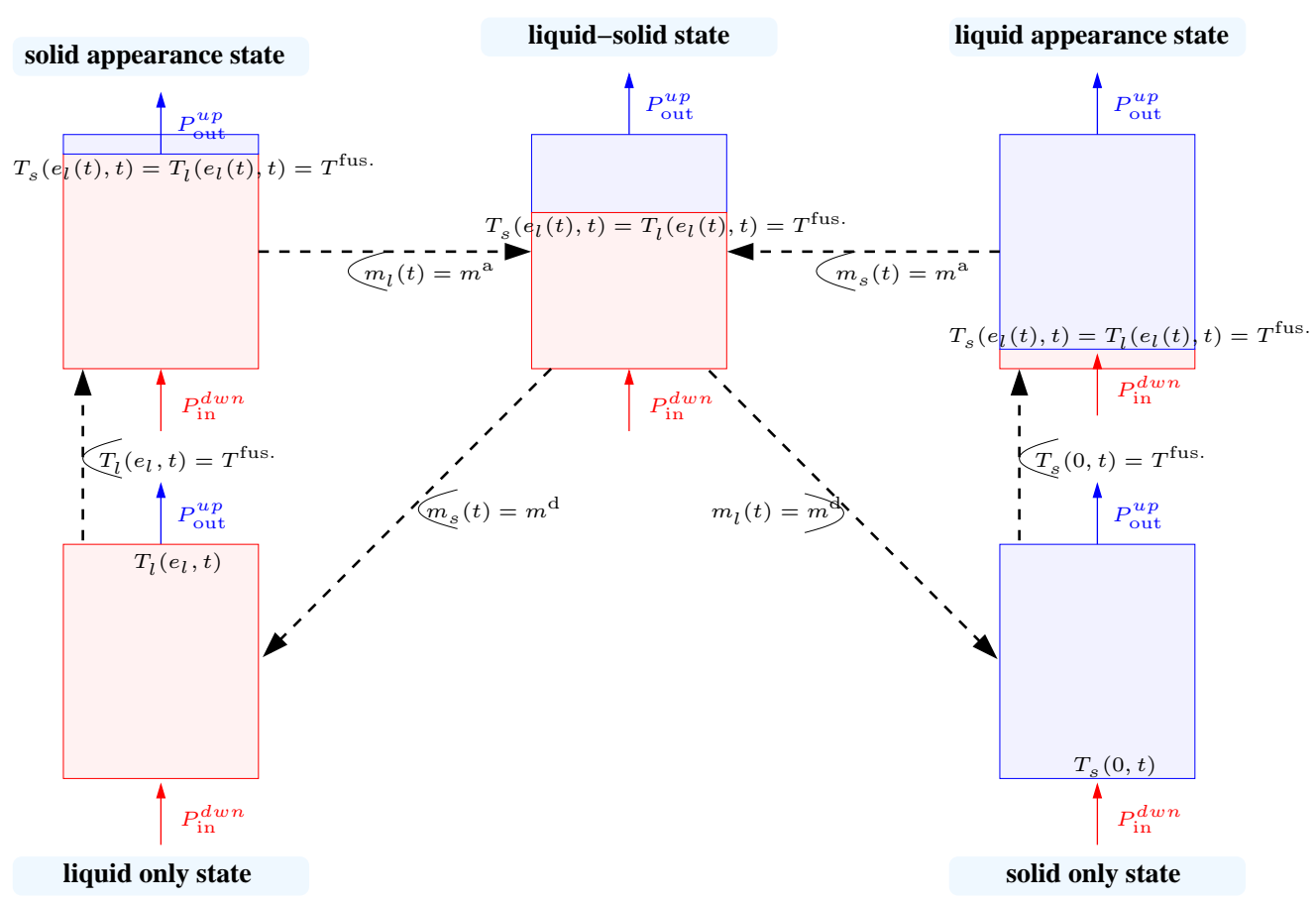

Notations (in addition to the ones already defined in Section 2):

$a(t)=b$
$\longrightarrow-\longrightarrow$ transition with associated condition $a(t)=b$;

$m^{a}$ (resp. $m^{d}$ ) mass threshold associated to a phase appearance (resp. disappearance);

$P_{\text {out }}^{u p}$ power exchanged by the system at its upper boundary;

$P_{\text {in }}^{d w n}$ power received by the system at its lower boundary.

Figure A.10: ODE system states and transitions. 


\section{References}

[1] H. Hu, S. A. Argyropoulos, Mathematical modelling of solidification and melting: a review, Modelling and Simulation in Materials Science and Engineering 4 (4) (1996) 371-396. doi:10.1088/0965-0393/4/4/004

[2] V. R. Voller, An overview of numerical methods for solving phase change problems, Advances in numerical heat transfer 1 (9) (1997) 341-380.

[3] E. Assis, L. Katsman, G. Ziskind, R. Letan, Numerical and experimental study of melting in a spherical shell, International Journal of Heat and Mass Transfer 50 (9-10) (2007) 1790-1804. doi:10.1016/j.ijheatmasstransfer.2006.10.007

[4] S. Wang, A. Faghri, B. T. L., A comprehensive numerical model for melting with natural convection, International Journal of Heat and Mass Transfer 53 (2010) 1986-2000.

[5] C. An, J. Su, Lumped parameter model for one-dimensional melting in a slab with volumetric heat generation, Applied Thermal Engineering 60 (12) (2013) 387-396. doi:10.1016/j . applthermaleng. 2013.07.018

[6] J. Crepeau, A. Siahpush, Approximate solutions to the Stefan problem with internal heat generation, Heat and Mass Transfer 44 (7) (2008) 787-794. doi:10.1007/s00231-007-0298-8.

[7] J. C. Crepeau, A. Siahpush, B. Spotten, On the Stefan problem with volumetric energy generation, Heat and Mass Transfer 46 (1) (2009) 119-128. doi:10.1007/s00231-009-0550-5.

[8] J. M. Bonnet, J. M. Seiler, Thermohydraulic phenomena in corium pool: the bali experiment, in: Proc. of ICONE 7, Tokyo, Japan, 1999.

[9] R. Le Tellier, L. Saas, F. Payot, Phenomenological analyses of corium propagation in LWRs: the PROCOR software platform, in: Proc. of the 7th 
European Review Meeting on Severe Accident Research ERMSAR-2015, Marseille, France, 2015.

[10] G. Carey, Derivative calculation from finite element solutions, Computer Methods in Applied Mechanics and Engineering 35 (1982) 1-14.

[11] Fauske \& Associates Inc., MAAP4 - modular accident analysis program for LWR power plants, in: Code Structure and Theory, Vol. 2, Electric Power Research Institute, 1994, Ch. 1.

[12] G. M. Phillips, Explicit forms for certain hermite approximations, BIT Numerical Mathematics 13 (2) (1973) 177-180. doi:10.1007/BF01933490

[13] J. Seiler, B. Tourniaire, A phenomenological analysis of melt progression in the lower head of a pressurized water reactor, Nuclear Engineering and Design 268 (2014) $87-95$.

[14] R. Le Tellier, L. Saas, S. Bajard, Transient stratification modelling of a corium pool in a LWR vessel lower head, Nuclear Engineering and Design 287 (2015) 68-77.

[15] B. Spindler, B. Tourniaire, J. M. Seiler, Simulation of MCCI with the TOLBIAC-ICB code based on the phase segregation model, Nuclear Engineering and Design 236 (2006) 2264-2270.

[16] J. L. Rempe, D. L. Knudson, M. Cebull, C. L. Atwood, Potential for invessel retention through ex- vessel flooding, in: Proc. of the OECD/CSNI Workshop on In-Vessel Core Debris Retention and Coolability, 1998.

[17] Apache Software Foundation, Apache commons math, release 3.6.1 (2016). URL http://commons . apache.org/math

${ }_{535}[18]$ G. K. Gupta, R. Sacks-Davis, P. E. Tischer, A review of recent developments in solving ODEs, Computing Surveys 17 (1) (1985) 5-47. 